\title{
Really Straight Graph Drawings ${ }^{\star}$
}

\author{
Vida Dujmović ${ }^{1,2}$, Matthew Suderman ${ }^{1}$, and David R. $\operatorname{Wood}^{2,3}$ \\ 1 School of Computer Science, McGill University, Montréal, Canada \\ \{vida, suderman\}@cs.mcgill.ca \\ 2 School of Computer Science, Carleton University, Ottawa, Canada \\ davidw@scs.carleton.ca \\ 3 Department of Applied Mathematics, Charles University, Prague, Czech Republic
}

\begin{abstract}
We study straight-line drawings of graphs with few segments and few slopes. Optimal results are obtained for all trees. Tight bounds are obtained for outerplanar graphs, 2-trees, and planar 3-trees. We prove that every 3 -connected plane graph on $n$ vertices has a plane drawing with at most $5 n / 2$ segments and at most $2 n$ slopes, and that every cubic 3 -connected plane graph has a plane drawing with three slopes (and three bends on the outerface). Drawings of non-planar graphs with few slopes are also considered. For example, it is proved that graphs of bounded degree and bounded treewidth have drawings with $\mathcal{O}(\log n)$ slopes.
\end{abstract}

\section{Introduction}

A common requirement for an aesthetically pleasing drawing of graph is that the edges are straight. This paper studies the following additional requirements of straight-line graph drawings:

1. minimise the number of segments in the drawing

2. minimise the number of distinct edge slopes in the drawing

First we formalise these notions. Consider a mapping of the vertices of a graph to distinct points in the plane. Now represent each edge by the closed line segment between its endpoints. Such a mapping is a (straight-line) drawing if each edge does not intersect any vertex, except for its own endpoints. By a segment in a drawing, we mean a maximal set of edges that form a line segment. The slope of a line $L$ is the angle swept from the $\mathrm{X}$-axis in an anticlockwise direction to $L$ (and is thus in $[0, \pi)$ ). The slope of an edge or segment is the slope of the line that extends it. A crossing in a drawing is a pair of edges that intersect at some point other than a common endpoint. A drawing is plane if it has no crossings. A plane graph is a planar graph with a fixed combinatorial embedding and a specified outerface. We emphasise that a plane drawing of a plane graph must preserve the embedding and outerface. That every plane graph has a plane drawing is a classical result independently due to Wagner and Fáry.

\footnotetext{
* Research initiated at the International Workshop on Fixed Parameter Tractability in Geometry and Games, organised by Sue Whitesides; Bellairs Research Institute of McGill University, Holetown, Barbados, Feb. 7-13, 2004. Research supported by NSERC and COMBSTRU.
} 
It is easily seen that a graph has a (plane) drawing on two slopes if and only if it has a (plane) drawing on any two slopes [3]. Garg and Tamassia [8] proved that it is $\mathcal{N} \mathcal{P}$-complete to decide whether a graph has a rectilinear planar drawing (that is, with vertical and horizontal edges). Thus it is $\mathcal{N} \mathcal{P}$-complete to decide whether a graph has a plane drawing with two slopes.

Our results include lower and upper bounds on the minimum number of segments and slopes in plane drawings of graphs, as summarised in Table 1. Due to space limitations, a number of auxiliary results and most proofs are omitted from this paper; see [3] for all the details. We refer the reader to the survey of Bodlaender [1] for the definition of treewidth, pathwidth, and $k$-tree.

First observe that the minimum number of slopes in a drawing of (plane) graph $G$ is at most the minimum number of segments in a drawing of $G$. Upper bounds for plane graphs are stronger than for planar graphs, since for planar graphs one has the freedom to choose the embedding and outerface. On the other hand, lower bounds for planar graphs are stronger than for plane graphs. For example, consider the $n$-vertex planar triangulation illustrated in Figure 1 . It has at least $n+2$ slopes in every plane drawing. Now fix the outerface to that illustrated in (a). Then there are at least $2 n-2$ slopes in every plane drawing. However, using the embedding shown in (b), there is a plane drawing with only $\lceil 3 n / 2\rceil$ slopes.

Section 2 studies plane drawings of 3-connected plane and planar graphs. In the case of slope-minimisation for plane graphs we obtain a bound that is tight in the worst case. However, our lower bound examples have linear maximum degree. In Section 3 we (drastically) improve this result in the case of cubic graphs, by proving that every 3-connected plane cubic graph has a plane drawing with three slopes, except for three edges on the outerface that have their own slope. As a corollary we prove that every 3-connected plane cubic graph has a plane 'drawing' with three slopes and three bends on the outerface. Section 4 considers non-plane drawings of arbitrary graphs with few slopes. For example, we prove that every graph with bounded degree and bounded treewidth has a drawing with $\mathcal{O}(\log n)$ slopes.

Before continuing, we outline some related research from the literature.

- Eppstein [6] characterised those planar graphs that have plane drawings with a segment between every pair of vertices. In some sense, these are the plane drawings with the least number of slopes.

- The geometric thickness of a graph $G$ is the minimum $k$ such that $G$ has a drawing in which every edge receives one of $k$ colours, and monochromatic



(a)

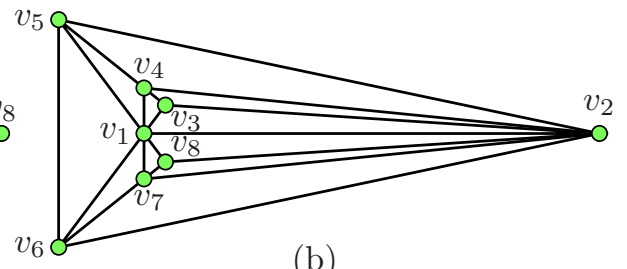

(b)

Fig. 1. 
Table 1. Summary of results (ignoring additive constants). Here $n$ is the number of vertices, $\eta$ is the number of vertices of odd degree, and $\Delta$ is the maximum degree. The lower bounds are existential, except for trees, for which the lower bounds are universal.

\begin{tabular}{lcccc}
\hline graph family & \multicolumn{2}{c}{ \# segments } & \multicolumn{2}{c}{ \# slopes } \\
& $\geq$ & $\leq$ & $\geq$ & $\leq$ \\
\hline trees & $\eta / 2$ & $\eta / 2$ & $\lceil\Delta / 2\rceil$ & $\lceil\Delta / 2\rceil$ \\
maximal outerplanar & $n$ & $n$ & - & $n$ \\
plane 2-trees & $2 n$ & $2 n$ & $2 n$ & $2 n$ \\
plane 3-trees & $2 n$ & $2 n$ & $2 n$ & $2 n$ \\
plane 2-connected & $5 n / 2$ & - & $2 n$ & - \\
planar 2-connected & $2 n$ & - & $n$ & - \\
plane 3-connected & $2 n$ & $5 n / 2$ & $2 n$ & $2 n$ \\
planar 3-connected & $2 n$ & $5 n / 2$ & $n$ & $2 n$ \\
plane 3-connected cubic & - & $n+2$ & 3 & 3 \\
\hline
\end{tabular}

edges do not cross (see $[5,7])$. In any drawing, edges with the same slope do not cross. Thus the geometric thickness of $G$ is a lower bound on the minimum number of slopes in a drawing of $G$.

- A drawing is convex if all the vertices are on the convex hull, and no three vertices are collinear. The book thickness of a graph (also called pagenumber and stacknumber) is the same as geometric thickness except that the drawing must be convex (see [4] for numerous references). Since edges with the same slope do not cross, the book thickness of $G$ is a lower bound on the minimum number of slopes in a convex drawing of $G$.

- Plane orthogonal drawings with two slopes (and few bends) have been extensively studied (see [12]). For example, Ungar [14] proved that every cyclically 4-edge-connected plane cubic graph has a plane drawing with two slopes and four bends on the outerface. Thus our above-mentioned result for 3connected plane cubic graphs nicely complements this theorem of Ungar.

- A drawing of the complete graph $K_{n}$ is defined by a set of $n$ points with no three collinear. Jamison [9] proved that the minimum number of slopes in a drawing of $K_{n}$ is $n$. The upper bound is obtained by positioning the vertices of $K_{n}$ on the vertices of a regular $n$-gon, as illustrated in Figure 2(a) and (b). In fact, Jamison [9] proved that every drawing of $K_{n}$ with exactly $n$ slopes is affinely equivalent to a regular $n$-gon. In [3] we study drawings of complete multi-partite graphs. For example, we prove that the minimum number of slopes in a convex drawing of $K_{n, n}$ is $n$, as illustrated in Figure 2(c).

- Wade and Chu [15] recognised that drawing arbitrary graphs with few slopes is an interesting problem. They defined the slope-number of a graph $G$ to be the minimum number of slopes in a drawing of $G$. However, the results of Wade and Chu only pertain to $K_{n}$. Seemingly unaware of the earlier work of Scott and Jamison, they rediscovered that the minimum number of slopes in a drawing of $K_{n}$ is $n$. In addition, they presented an algorithm to test if $K_{n}$ can be drawn using a given set of slopes. 


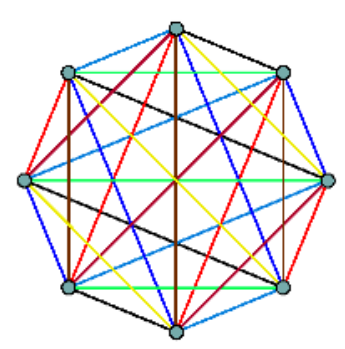

(a) $K_{r}(n$ even)

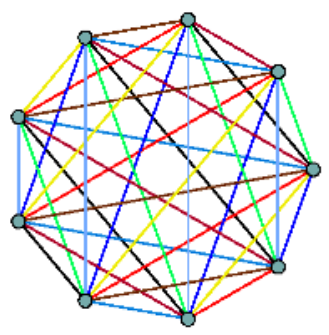

(b) $K_{r}(n$ odd $)$

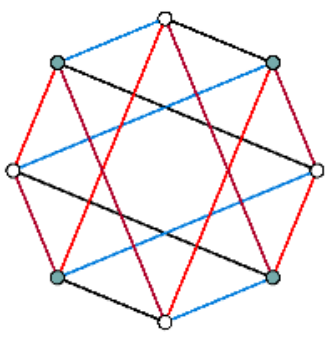

(c) $K_{n, n}$

Fig. 2. Drawings of $K_{n}$ and $K_{n, n}$ with $n$ slopes.

\section{3-Connected Plane Graphs}

Theorem 1. Every 3-connected plane graph with $n$ vertices has a plane drawing with at most $5 n / 2-3$ segments and at most $2 n-10$ slopes.

The proof of Theorem 1 is based on the canonical ordering of Kant [10]. Let $G$ be a 3 -connected plane graph. Kant [10] proved that $G$ has a canonical ordering defined as follows. Let $\sigma=\left(V_{1}, V_{2}, \ldots, V_{K}\right)$ be an ordered partition of $V(G)$. That is, $V_{1} \cup V_{2} \cup \cdots \cup V_{K}=V(G)$ and $V_{i} \cap V_{j}=\emptyset$ for all $i \neq j$. Define $G_{i}$ to be the plane subgraph of $G$ induced by $V_{1} \cup V_{2} \cup \cdots \cup V_{i}$. Let $C_{i}$ be the subgraph of $G$ induced by the edges on the boundary of the outerface of $G_{i}$. Then $\sigma$ is a canonical ordering of $G$ if:

- $V_{1}=\left\{v_{1}, v_{2}\right\}$, where $v_{1}$ and $v_{2}$ lie on the outerface and $v_{1} v_{2} \in E(G)$.

- $V_{K}=\left\{v_{n}\right\}$, where $v_{n}$ lies on the outerface, $v_{1} v_{n} \in E(G)$, and $v_{n} \neq v_{2}$.

- Each $C_{i}(i>1)$ is a cycle containing $v_{1} v_{2}$.

- Each $G_{i}$ is biconnected and internally 3-connected; that is, removing any two interior vertices of $G_{i}$ does not disconnect it.

- For each $i \in\{2,3, \ldots, K-1\}$, one of the following condition holds:

1. $V_{i}=\left\{v_{i}\right\}$ where $v_{i}$ is a vertex of $C_{i}$ with at least three neighbours in $C_{i-1}$, and $v_{i}$ has at least one neighbour in $G \backslash G_{i}$.

2. $V_{i}=\left(s_{1}, s_{2}, \ldots, s_{\ell}, v_{i}\right), \ell \geq 0$, is a path in $C_{i}$, where each vertex in $V_{i}$ has at least one neighbour in $G \backslash G_{i}$. Furthermore, the first and the last vertex in $V_{i}$ have one neighbour in $C_{i-1}$, and these are the only two edges between $V_{i}$ and $G_{i-1}$.

The vertex $v_{i}$ is called the representative vertex of $V_{i}, 2 \leq i \leq K$. The vertices $\left\{s_{1}, s_{2}, \ldots, s_{\ell}\right\} \subseteq V_{i}$ are called division vertices. Let $S \subset V(G)$ be the set of all division vertices. A vertex $u$ is a successor of a vertex $w \in V_{i}$ if $u w$ is an edge and $u \in G \backslash G_{i}$, and $u$ is a predecessor of $w \in V_{i}$ if $u w$ is an edge and $u \in V_{j}$ for some $j<i$. We also say that $u$ is a predecessor of $V_{i}$. Let $P\left(V_{i}\right)=\left(p_{1}, p_{2}, \ldots, p_{q}\right)$ be the set of predecessors of $V_{i}$ ordered by the path from $v_{1}$ to $v_{2}$ in $C_{i-1} \backslash v_{1} v_{2}$. Vertex $p_{1}$ and $p_{q}$ are the left and right predecessors of $V_{i}$ respectively, and vertices $p_{2}, p_{3}, \ldots p_{q-1}$ are called middle predecessors of $V_{i}$. 
Theorem 2. Let $\sigma$ be a canonical ordering of an $n$-vertex $m$-edge plane 3connected graph $G$. Define $S$ as above. Then $G$ has a plane drawing $D$ with at most $m-\max \{\lceil n / 2\rceil-|S|-3,|S|\}$ segments, and at most $m-\max \{n-|S|-4$, $|S|\}$ slopes.

Proof Construction. For every vertex $v$, let $X(v)$ and $Y(v)$ denote the $x$ and $y$ coordinates of $v$, respectively. If a vertex $v$ has a neighbour $w$, such that $X(w)<X(v)$ and $Y(w)<Y(v)$, then we say $v w$ is a left edge of $v$. Similarly, if $v$ has a neighbour $w$, such that $X(w)>X(v)$ and $Y(w)<Y(v)$, then we say $v w$ is a right edge of $v$. If $v w$ is an edge such that $X(v)=X(w)$ and $Y(v)<Y(w)$, than we say $v w$ is a vertical edge above $v$ and below $w$.

We define $D$ inductively on $\sigma=\left(V_{1}, V_{2}, \ldots, V_{K}\right)$ as follows. Let $D_{i}$ denote a drawing of $G_{i}$. A vertex $v$ is a peak in $D_{i}$, if each neighbour $w$ of $v$ has $Y(w) \leq Y(v)$ in $D_{i}$. We say that a point $p$ in the plane is visible in $D_{i}$ from vertex $v \in D_{i}$, if the segment $\overline{p v}$ does not intersect $D_{i}$ except at $v$. At the $i^{\text {th }}$ induction step, $2 \leq i \leq K, D_{i}$ will satisfy the following invariants:

Invariant 1: $C_{i} \backslash v_{1} v_{2}$ is strictly $X$-monotone; that is, the path from $v_{1}$ to $v_{2}$ in $C_{i} \backslash v_{1} v_{2}$ has (strictly) increasing X-coordinates.

Invariant 2: Every peak in $D_{i}, i<K$, has a successor.

Invariant 3: Every representative vertex $v_{j} \in V_{j}, 2 \leq j \leq i$ has a left and a right edge. Moreover, if $\left|P\left(V_{j}\right)\right| \geq 3$ then there is a vertical edge below $v_{j}$.

Invariant 4: $D_{i}$ has no edge crossings.

For the base case $i=2$, position the vertices $v_{1}, v_{2}$ and $v_{3}$ at the corners of an equilateral triangle so that $X\left(v_{1}\right)<X\left(v_{3}\right)<X\left(v_{2}\right)$ and $Y\left(v_{1}\right)<Y\left(v_{2}\right)<$ $Y\left(v_{3}\right)$. Draw the division vertices of $V_{2}$ on the segment $v_{1} v_{3}$. This drawing of $D_{2}$ satisfies all four invariants. Now suppose that we have a drawing of $D_{i-1}$ that satisfies the invariants. There are two cases to consider in the construction of $D_{i}$, corresponding to the two cases in the definition of the canonical ordering.

Case 1. $\left|P\left(V_{i}\right)\right| \geq 3$ : If $v_{i}$ has a middle predecessor $v_{j}$ with $\left|P\left(V_{j}\right)\right| \geq 3$, let $w=v_{j}$. Otherwise let $w$ be any middle predecessor of $v_{i}$. Let $L$ be the open ray $\{(X(w), y): y>Y(w)\}$. By invariant 1 for $D_{i-1}$, there is a point in $L$ that is visible in $D_{i-1}$ from every predecessor of $v_{i}$. Represent $v_{i}$ by such a point, and draw segments between $v_{i}$ and each of its predecessors. That the resulting drawing $D_{i}$ satisfies the four invariants can be immediately verified.

Case 2. $\left|P\left(V_{i}\right)\right|=2$ : Suppose that $P\left(V_{i}\right)=\{w, u\}$, where $w$ and $u$ are the left and the right predecessors of $V_{i}$, respectively. Suppose $Y(w) \geq Y(u)$. (The other case is symmetric.) Let $P$ be the path between $w$ and $u$ on $C_{i-1} \backslash v_{1} v_{2}$. As illustrated in Figure 3, let $A_{i}$ be the region $\{(x, y): y>Y(w)$ and $X(w) \leq$ $x \leq X(u)\}$. Assume on the contrary that $D_{i-1} \cap A_{i} \neq \emptyset$. By the monotonicity of $D_{i-1}, P \cap A_{i} \neq \emptyset$. Let $p \in P \cap A_{i}$. Since $Y(p)>Y(w) \geq Y(u), P$ is $X$-monotone and thus has a vertex between $w$ and $u$ that is a peak. By the definition of the canonical ordering $\sigma$, the addition of $V_{i}$ creates a face of $G$, since $V_{i}$ is added in the outerface of $G_{i-1}$. Therefore, each vertex between $w$ and $u$ on $P$ has no successor, and is thus not a peak in $D_{i-1}$ by invariant 2, which is the desired contradiction. Therefore $D_{i-1} \cap A_{i}=\emptyset$. 


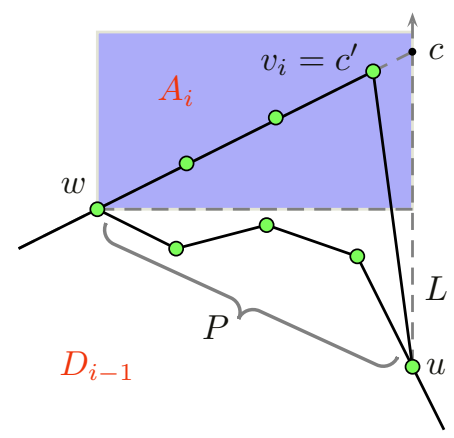

Fig. 3. Illustration for Case 2.

Let $L$ be the open ray $\{(X(u), y): y>Y(u)\}$. If $w \notin S$, then by invariant 3 , $w$ has a left and a right edge in $D_{i-1}$. Let $c$ be the point of intersection between $L$ and the line extending the left edge at $w$. If $w \in S$, then let $c$ be any point in $A_{i}$ on $L$. By invariant 1 , there is a point $c^{\prime} \notin\{c, w\}$ on $\overline{w c}$ such that $c^{\prime}$ is visible in $D_{i-1}$ from $u$. Represent $v_{i}$ by $c^{\prime}$, and draw two segments $\overline{v_{i} u}$ and $\overline{v_{i} w}$. These two segments do not intersect any part of $D_{i-1}$ (and neither is horizontal). Represent any division vertices in $V_{i}$ by arbitrary points on the open segment $\overline{w v_{i} \cap A_{i}}$. Therefore, in the resulting drawing $D_{i}$, there are no crossings and the remaining three invariants are maintained. This completes the construction of $D$. The analysis for the number of segments and slopes is in [3].

Proof (of Theorem 1). Whenever a set $V_{i}$ is added to $G_{i-1}$, at least $\left|V_{i}\right|-1$ edges that are not in $G$ can be added so that the resulting graph is planar. Thus $|S|=\sum_{i}\left(\left|V_{i}\right|-1\right) \leq 3 n-6-m$. Hence Theorem 2 implies that $G$ has a plane drawing with at most $m-n / 2+|S|+3 \leq 5 n / 2-3$ segments, and at most $m-n+|S|-4 \leq 2 n-10$ slopes.

Since deleting an edge from a drawing cannot increase the number of slopes, and every plane graph can be triangulated to a 3-connected plane graph, Theorem 1 implies that every $n$-vertex plane graph has a plane drawing with at most $2 n-10$ slopes. Note that we cannot draw the same conclusion for segments, since deleting an edge in a drawing may increase the number of segments. The famous 'nested-triangles' planar graph leads to the following lower bound.

Lemma 1. For all $n \equiv 0(\bmod 3)$, there is an $n$-vertex planar triangulation with maximum degree six that has at least $2 n-6$ segments in every plane drawing, regardless of the choice of outerface.

\section{Cubic 3-Connected Plane Graphs}

A graph in which every vertex has degree three is cubic.

Theorem 3. Every cubic 3-connected plane graph has a plane drawing in which every edge has slope in $\{\pi / 4, \pi / 2,3 \pi / 4\}$, except for three edges on the outerface. 
Proof. Let $\sigma=\left(V_{1}, V_{2}, \ldots, V_{K}\right)$ be a canonical ordering of $G$. We re-use the notation from Theorem 2, except that a representative vertex of $V_{i}$ may be the first or last vertex in $V_{i}$. Since $G$ is cubic, $\left|P\left(V_{i}\right)\right|=2$ for all $1<i<K$, and every vertex not in $\left\{v_{1}, v_{2}, v_{n}\right\}$ has exactly one successor. We proceed by induction on $i$ with the hypothesis that $G_{i}$ has a plane drawing $D_{i}$ that satisfies:

Invariant 1: $C_{i} \backslash v_{1} v_{2}$ is $X$-monotone; that is, the path from $v_{1}$ to $v_{2}$ in $C_{i} \backslash v_{1} v_{2}$ has non-decreasing $\mathrm{X}$-coordinates.

Invariant 2: Every peak in $D_{i}, i<K$, has a successor.

Invariant 3: If there is a vertical edge above $v$ in $D_{i}$, then all the edges of $G$ that are incident to $v$ are in $G_{i}$.

Invariant 4: $D_{i}$ has no edge crossings.

Let $D_{2}$ be the drawing of $G_{2}$ constructed as follows. Draw $v_{1} v_{2}$ horizontally with $X\left(v_{1}\right)<X\left(v_{2}\right)$. This accounts for one edge whose slope is not in $\{\pi / 4, \pi / 2,3 \pi / 4\}$. Now draw $v_{1} v_{3}$ with slope $\pi / 4$, and draw $v_{2} v_{3}$ with slope $3 \pi / 4$. Add any division vertices on the segment $v_{1} v_{3}$. Now $v_{3}$ is the only peak in $D_{2}$, and it has a successor by the definition of the canonical ordering. Thus all the invariants are satisfied for the base case $D_{2}$.

Now suppose that $2<i<K$ and we have a drawing of $D_{i-1}$ that satisfies the invariants. Suppose that $P\left(V_{i}\right)=\{u, w\}$, where $u$ and $w$ are the left and the right predecessors of $V_{i}$, respectively. Without loss of generality, $Y(w) \leq Y(u)$. Let the representative vertex $v_{i}$ be last vertex in $V_{i}$. Position $v_{i}$ at the intersection of a vertical segment above $w$, and a segment of slope $\pi / 4$ from $u$, and add any division vertices on $\overline{u v_{i}}$, as illustrated in Figure 4(a). Note that there is no vertical edge above $w$ by invariant 3 for $D_{i-1}$. (For the case in which $Y(u)<Y(w)$, we take the representative vertex $v_{i}$ to be the first vertex in $V_{i}$, and the edge $w v_{i}$ has slope $3 \pi / 4$, as illustrated in Figure 4(b).)

Clearly the resulting drawing $D_{i}$ is $X$-monotone. Thus invariant 1 is maintained. The vertex $v_{i}$ is the only peak in $D_{i}$ that is not a peak in $D_{i-1}$. Since $v_{i}$ has a successor by the definition of the canonical ordering, invariant 2 is maintained. The vertical edge $w v_{i}$ satisfies invariant 3 , since $v_{i}$ is the sole successor of $w$. Thus invariant 3 is maintained. No vertex between $u$ and $w$ (on the path from $u$ to $w$ in $\left.C_{i-1} \backslash v_{1} v_{2}\right)$ is higher than the higher of $u$ and $w$. Otherwise there would be a peak, not equal to $v_{n}$, with no successor, and thus violating invariant 2 for $D_{i-1}$. Thus the edges in $D_{i} \backslash D_{i-1}$ do not cross any edges in $D_{i}$. In particular, there is no edge $u x$ in $D_{i-1}$ with slope $\pi / 4$ and $Y(x)>Y(u)$. The vertex $v_{n}$ can be easily added to the drawing to complete the construction.

(a)

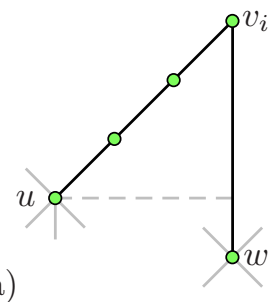



Fig. 4. Construction of a 3-slope drawing of a cubic 3-connected plane graph. 
It is easily seen that the bound of six on the number of slopes in Theorem 3 is optimal for any 3-connected cubic plane graph whose outerface is a triangle. An easy variation on the algorithm in Theorem 3 gives:

Corollary 1. Every cubic 3-connected plane graph has a plane 'drawing' with three slopes and three bends on the outerface.

\section{Drawings of General Graphs with Few Slopes}

This section is motivated by the following fundamental open problem: Is there a function $f$ such that every graph with maximum degree $\Delta$ has a drawing with at most $f(\Delta)$ slopes? This is open even for $\Delta=3$. Note that:

- The best lower bound that we are aware of is $\Delta+1$ for the complete graph.

- There is no such function $f$ for convex drawings. Malitz [11] proved that there are $\Delta$-regular $n$-vertex graphs with book thickness $\Omega\left(\sqrt{\Delta} n^{1 / 2-1 / \Delta}\right)$. Since book thickness is a lower bound on the number of slopes in a convex drawing, every convex drawing of such a graph has $\Omega\left(\sqrt{\Delta} n^{1 / 2-1 / \Delta}\right)$ slopes.

- An affirmative solution to this problem would imply that geometric thickness is bounded by maximum degree, which is an open problem due to Eppstein [7]. Duncan et al. [5] recently proved that graphs with maximum degree at most four have geometric thickness at most two.

Let $H$ be a (host) graph. The vertices of $H$ are called nodes. An $H$-partition of a graph $G$ is a function $f: V(G) \rightarrow V(H)$ such that for every edge $v w \in E(G)$ we have $f(v)=f(w)$ or $f(v) f(w) \in E(H)$. In the latter case, we say $v w$ is mapped to the edge $f(v) f(w)$. The width of $f$ is the maximum of $\left|f^{-1}(x)\right|$, taken over all nodes $x \in V(H)$, where $f^{-1}(x)=\{v \in V(G): f(v)=x\}$. In the following result, we describe how to produce a drawing of a graph $G$ given an $H$-partition of $G$ and a drawing $D$ of $H$. The general approach is to scale $D$ appropriately, and then replace each node of $H$ by a copy of the drawing of $K_{k}$ on a regular $k$-gon. The only difficulty is to scale $D$ so that we obtain a valid drawing of $G$.

Lemma 2 ([3]). Let $H$ be a graph admitting a drawing $D$ with $s$ distinct slopes and $\ell$ distinct edge lengths. Let $G$ be a graph admitting an $H$-partition of width $k$. Then $G$ has a drawing with $k s \ell(k-1)+k+s$ slopes.

Lemma 2 suggests looking at host graphs that admit drawings with few slopes and few edge lengths. Obviously a path has a drawing with one slope and one edge length. Based on this idea, we prove that every graph with bandwidth $b$ has a drawing with at most $\frac{1}{2} b(b+1)+1$ slopes. Based on results from the literature that bound bandwidth in terms of maximum degree $\Delta$, we conclude:

- Every interval graph has a drawing with at most $\frac{1}{2} \Delta(\Delta+1)+1$ slopes.

- Every co-comparability graph (which includes the permutation graphs) has a drawing with at most $\Delta(2 \Delta-1)+1$ slopes.

- Every AT-free graph has a drawing with at most $\frac{3}{2} \Delta(3 \Delta+1)+1$ slopes. 
Lemma 2 motivates the study of drawings of trees with few slopes and few distinct edge lengths.

Lemma 3. Every tree $T$ with pathwidth $k \geq 1$ has a plane drawing with $\max \{\Delta(T)-1,1\}$ slopes and $2 k-1$ distinct edge lengths.

Lemma 4 ([13]). Every tree $T$ has a path $P$, called a "backbone", such that $T \backslash V(P)$ has smaller pathwidth than $T$, and the endpoints of $P$ are leaves of $T$.

Proof (of Lemma 3). We refer to $T$ as $T_{0}$. Let $n_{0}$ be the number of vertices in $T_{0}$, and let $\Delta_{0}=\Delta\left(T_{0}\right)$. The result holds trivially for $\Delta_{0} \leq 2$. Now assume that $\Delta_{0} \geq 3$. Let $S$ be the set of slopes $S=\left\{\frac{\pi}{2}\left(1+\frac{i}{\Delta_{0}-2}\right): 0 \leq i \leq \Delta_{0}-2\right\}$. We proceed by induction on $n$ with the hypothesis: "There is a real number $\ell=\ell\left(n_{0}, \Delta_{0}\right)$, such that for every tree $T$ with $n \leq n_{0}$ vertices, maximum degree at most $\Delta_{0}$, and pathwidth $k \geq 1$, and for every vertex $r$ of $T$ with degree less than $\Delta_{0}, T$ has a plane drawing $D$ in which:

- $r$ is at the top of $D$ (that is, no point in $D$ has greater Y-coordinate than $r$ ),

- every edge of $T$ has slope in $S$,

- every edge of $T$ has length in $\left\{\ell^{0}, \ell^{1}, \ldots, \ell^{2 k-1}\right\}$, and

- if $r$ is contained in some backbone of $T$, then every edge of $T$ has length in $\left\{\ell^{0}, \ell^{1}, \ldots, \ell^{2 k-2}\right\} . "$

The result follows from the induction hypothesis, since we can take $r$ to be the endpoint of a backbone of $T_{0}$, in which case $\operatorname{deg}(r)=1<\Delta_{0}$, and thus every edge of $T_{0}$ has length in $\left\{\ell^{0}, \ell^{1}, \ldots, \ell^{2 k-2}\right\}$.

The base case with $n=1$ is trivial. Now suppose that the hypothesis is true for trees on less than $n$ vertices, and we are given a tree $T$ with $n$ vertices and pathwidth $k$, and $r$ is a vertex of $T$ with degree less than $\Delta_{0}$.

If $r$ is contained in some backbone $B$ of $T$, then let $P=B$. Otherwise, let $P$ be a path from $r$ to an endpoint of a backbone $B$ of $T$. Note that $P$ has at least one edge. As illustrated in Figure 5, draw $P$ horizontally with unit-length edges. Every vertex in $P$ has at most $\Delta_{0}-2$ neighbours in $T \backslash V(P)$, since $r$ has degree less than $\Delta_{0}$ and the endpoints of a backbone are leaves. At each vertex $x \in P$, the children $\left\{y_{0}, y_{1}, \ldots, y_{\Delta_{0}-3}\right\}$ of $x$ are positioned below $P$ and on the unit-circle centred at $x$, so that each edge $x y_{j}$ has slope $\frac{\pi}{2}\left(1+j /\left(\Delta_{0}-2\right)\right) \in S$.

Every connected component $T^{\prime}$ of $T \backslash V(P)$ is a tree rooted at some vertex $r^{\prime}$ adjacent to a vertex in $P$. Thus $r^{\prime}$ has already been positioned in the drawing of $T$. If $T^{\prime}$ is a single vertex, then we no longer need to consider this $T^{\prime}$.

We consider two types of subtrees $T^{\prime}$, depending on whether the pathwidth of $T^{\prime}$ is less than $k$. Suppose that the pathwidth of $T^{\prime}$ is $k$ (it cannot be more). Then $T^{\prime} \cap B \neq \emptyset$ since $B$ is a backbone of $T$. Thus $T^{\prime} \cap B$ is a backbone of $T^{\prime}$ containing $r^{\prime}$. Thus we can apply the stronger induction hypothesis in this case.

Every $T^{\prime}$ has less vertices than $T$, and every $r^{\prime}$ has degree less than $\Delta_{0}$ in $T^{\prime}$. Thus by induction, every $T^{\prime}$ has a drawing with $r^{\prime}$ at the top, and every edge of $T^{\prime}$ has slope in $S$. Furthermore, if the pathwidth of $T^{\prime}$ is less than $k$, then every edge of $T^{\prime}$ has length in $\left\{\ell^{0}, \ell^{1}, \ldots, \ell^{2 k-3}\right\}$. Otherwise $r^{\prime}$ is in a backbone of $T^{\prime}$, and every edge of $T^{\prime}$ has length in $\left\{\ell^{0}, \ell^{1}, \ldots, \ell^{2 k-2}\right\}$. 


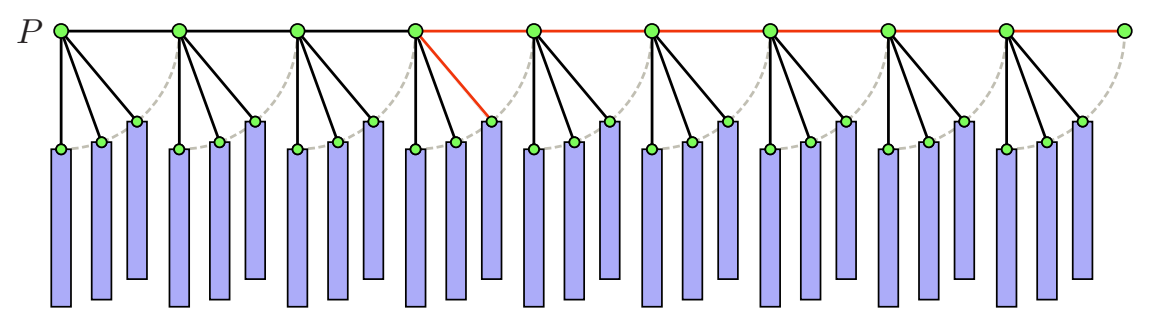

Fig. 5. Drawing of $T$ with few slopes and few edge lengths.

There exists a scale factor $\ell<1$, depending only on $n_{0}$ and $\Delta_{0}$, so that by scaling the drawings of every $T^{\prime}$ by $\ell$, the widths of the drawings are small enough so that there is no crossings when the drawings are positioned with each $r^{\prime}$ at its already chosen location. (Note that $\ell$ is the same value at every level of the induction.) Scaling preserves the slopes of the edges. An edge in any $T^{\prime}$ that had length $\ell^{i}$ before scaling, now has length $\ell^{i+1}$.

Case 1. $r$ is contained in some backbone $B$ of $T$ : By construction, $P=B$. So every $T^{\prime}$ has pathwidth at most $k-1$, and thus every edge of $T^{\prime}$ has length in $\left\{\ell^{1}, \ell^{2}, \ldots, \ell^{2 k-2}\right\}$. All the other edges of $T$ have unit-length. Thus we have a plane drawing of $T$ with edge lengths $\left\{\ell^{0}, \ell^{1}, \ldots, \ell^{2 k-2}\right\}$, as claimed.

Case 2. $r$ is not contained in any backbone of $T$ : Every edge in every $T^{\prime}$ has length in $\left\{\ell^{1}, \ell^{2}, \ldots, \ell^{2 k-1}\right\}$. All the other edges of $T$ have unit-length. Thus we have a plane drawing of $T$ with edge lengths $\left\{\ell^{0}, \ell^{1}, \ldots, \ell^{2 k-1}\right\}$, as claimed.

Theorem 4. Let $G$ be a graph with $n$ vertices, maximum degree $\Delta$, and treewidth $k$. Then $G$ has a drawing with $\mathcal{O}\left(k^{3} \Delta^{4} \log n\right)$ slopes.

Proof. Ding and Oporowski [2] proved that for some tree $T, G$ has a $T$-partition of width at most $\max \{24 k \Delta, 1\}$. Let $w=\max \{24 k \Delta, 1\}$. For each node $x \in$ $V(T)$, there are at most $w \Delta$ edges of $G$ incident to vertices mapped to $x$. Hence we can assume that $T$ is a forest with maximum degree at most $w \Delta$, as otherwise there is an edge of $T$ with no edge of $G$ mapped to it, in which case the edge of $T$ can be deleted. Similarly, $T$ has at most $n$ vertices. Now, $T$ has pathwidth at most $\log (2 n+1)$ (see [1]). By Lemma 3, $T$ has a drawing with at most $w \Delta-1$ slopes and at most $2 \log (2 n+1)-1$ distinct edge lengths. By Lemma $2, G$ has a drawing in which the number of slopes is at most $w(w \Delta-1)(2 \log (2 n+1)-1)(w-1)+$ $(w \Delta-1)+w \in \mathcal{O}\left(w^{3} \Delta \log n\right) \subseteq \mathcal{O}\left(k^{3} \Delta^{4} \log n\right)$.

Corollary 2. Every n-vertex graph with bounded degree and bounded treewidth has a drawing with $\mathcal{O}(\log n)$ slopes.

\section{Acknowledgements}

Thanks to all of the participants of the Bellairs workshop for creating a stimulating working environment. Special thanks to Mike Fellows for suggesting the problem. 


\section{References}

1. Hans L. Bodlaender. A partial $k$-arboretum of graphs with bounded treewidth. Theoret. Comput. Sci., 209(1-2):1-45, 1998.

2. Guoli Ding and Bogdan Oporowski. Some results on tree decomposition of graphs. J. Graph Theory, 20(4):481-499, 1995.

3. Vida Dujmović, Matthew Suderman, and David R. Wood. Really straight graph drawings. arXiv.org:cs.DM/0405112, 2004.

4. Vida Dujmović And David R. Wood. On linear layouts of graphs. Discrete Math. Theor. Comput. Sci., 6(2):339-358, 2004.

5. Christian A. Duncan, David Eppstein, and Stephen G. Kobourov. The geometric thickness of low degree graphs. In Proc. 20th ACM Symp. on Computational Geometry (SoCG '04), pp. 340-346. ACM Press, 2004.

6. DAvid EPpstein. Dilation-free planar graphs. http://www.ics.uci.edu/ ${ }^{\sim}$ eppstein/junkyard/dilation-free/, 1997.

7. DAVID EPpstein. Separating thickness from geometric thickness. In JÁnOs PACH, editor, Towards a Theory of Geometric Graphs, vol. 342 of Contemporary Mathematics, pp. 75-86. Amer. Math. Soc., 2004.

8. Ashim Garg and Roberto Tamassia. On the computational complexity of upward and rectilinear planarity testing. SIAM J. Comput., 31(2):601-625, 2001.

9. Robert E. Jamison. Few slopes without collinearity. Discrete Math., 60:199-206, 1986.

10. Goos Kant. Drawing planar graphs using the canonical ordering. Algorithmica, 16(1):4-32, 1996.

11. Seth M. Malitz. Graphs with $E$ edges have pagenumber $O(\sqrt{E})$. J. Algorithms, 17(1):71-84, 1994.

12. Md. Saidur Rahman, Takao Nishizeki, and Shubhashis Ghosh. Rectangular drawings of planar graphs. J. Algorithms, 50:62-78, 2004.

13. Matthew Suderman. Pathwidth and layered drawings of trees. Internat. J. Comput. Geom. Appl., 14(3):203-225, 2004.

14. Peter Ungar. On diagrams representing maps. J. London Math. Soc., 28:336342, 1953.

15. Greg A. Wade and Jiang-Hsing Chu. Drawability of complete graphs using a minimal slope set. The Computer Journal, 37(2):139-142, 1994. 